\title{
Designing an energy performance rewards system based on the energy conservation mathematical model
}

\author{
Tsai Yung -Tse* \\ Department of Business Administration, Nanhua University, No.55, Sec. 1, Nanhua Rd., Dalin Township, Chiayi County 62249 , \\ Taiwan
}

\begin{abstract}
In this study, we design an energy conservation mathematical model toward the performance reward system to improve the nation to pay more attention to the few energy resources on earth. No concerning paper is similar to this topic. So this issue is getting more and more important. This issue is gaining increasing importance as a dwindling energy rate may potentially lead to no more energy resources. It is vitally important for authorities concerned to consider ways to improve the energy performance and maintain the economic size required for the whole national development. On the basis of the aforementioned discussion, the paper provides the background for mathematical modeling for a method by which decision makers will be able to measure the industry's' energy performance based on the energy conservation mathematical model made in the study. This model will serve multiple purposes, but among others, it can be applied to the design of an energy performance reward system in which the nation focuses on energy resources with either poorer or higher capacity.
\end{abstract}

Keywords: Energy performance reward system, energy conservation mathematical model, capacity

\section{Introduction}

Energy on Earth is getting less and less day by day. Taiwan is an island nation, this situation is obvious here. In the study, the government has responsibility to adopt an economic and total quality management which is primarily focused on the energy issue. [1] However, relatively fixed energy resources are also required in order to maintain the nation's economic size above a certain level, so how to decide an energy performance is an important issue now. [2] This can control the waste and use of energy for each industry. [3] There are currently a number of problems with the reducing distribution of energy resources. [4] In addition to the same issue such as the excessive emphasis placed on the waste use of energy is also neglected by us. In this study, we assume that rewards system part of the incentive for the energy authorities concerned to identify poorly performing industries and provide them with guidance. The mathematics modelling is a way to measure and decide the performance of the industries. [4]

\section{Mathematical Modelling}

For the reason that energy should be conserved for our generation. As a result, the nation and all industries should pay more attention to the energy crisis issue. The energy conservation mathematical model with the decision making environmental criteria of this mathematical model are as follows:

\subsection{Mathematical symbols \& assumptions}

$N$ The number of the industries in a nation.

$M$ The number of energy resources.

*Manuscript received December 8, 2015; revised April 3, 2016.

Corresponding author. Tel. +-886-5-2830630; E-mail address: mysteventsai@yahoo.com.tw.

doi: $10.12720 /$ sgce.5.2.129-134 
$z \quad$ The energy conservation of each industry. $z \in[0,1]$.

$\frac{x(z)}{z}$ The average value that represents the effort indicator value of an industry energy conservation potential $z .0 \leq x(z) \leq z$.

$C\left(\frac{x(z)}{z}\right)$ The cost if the nation wants the $z$-value industry to present an effort indicator value of:

$$
\frac{x(z)}{z}
$$

$\mathrm{H}$ The total budget for energy conservation performance rewards.

$r_{z}$ The "unit" of performance reward.

$f_{i}(z)$ The distribution and density function for a $z$-value industry in a nation $i, i=1.2 \ldots . . N$

$\mu_{i}$ The mean value of $f_{i}$.

$\sigma_{i}^{2}$ The variance of $f_{i}$.

$W_{z}$ The degree to which the government focuses on the $z$-value industry's energy conservation performance, where

$$
\int_{0}^{1} W_{z} d z=1
$$

Hence:

$$
W_{z}=w \cdot z+\left(1-\frac{w}{2}\right)
$$

$w$ is declared a known constant.

$n_{z}:$ The number of $z$-value industry.

\subsection{Mathematical models \& optimal solutions}

\subsubsection{Decision-Making model for the industries}

For $z$-value industry with energy conservation performance $x(z))$ is to maximize the total performance reward. The model can thus be written as [5]:

$$
\underset{0 \leq x(z) \leq z}{\operatorname{Max}} \int_{0}^{1}\left[r_{z} \cdot \frac{x(z)}{z}-C\left(\frac{x(z)}{z}\right)\right] \cdot M \cdot f_{i}(z) d z
$$

$x^{*}(z)$ is the optimal solution of (5). [1]

\subsubsection{Decision-Making model for the nation}

If the nation determines the $r_{z}$ value for each $z$, the reward that the nation issues to the industry $i$ will be: 
$\int_{0}^{1} r_{z} \cdot \frac{x^{*}(z)}{z} \cdot M \cdot f_{i}(z) d z$, where $\frac{x^{*}(z)}{z}=C^{\prime-1}\left(r_{z}\right) \quad \forall z$

In the meantime, the contribution value is

$$
\int_{0}^{1}\left[w z+\left(1-\frac{w}{2}\right)\right] \cdot C^{\prime-1}\left(r_{z}\right) \cdot M \cdot f_{i}(z) d z
$$

The mathematical model according to which the nation must determine $r_{z}$ for each $z$ without exceeding budget $\mathrm{H}$ in order to maximize energy conservation performance is:

$$
\left\{\begin{array}{l}
\underset{r}{\operatorname{Max}} \int_{0}^{1}\left[w z+\left(1-\frac{w}{2}\right)\right] \cdot C^{\prime-1}\left(r_{z}\right) \cdot M \cdot n_{z} d z \\
\text { s.t. } \int_{0}^{1} n_{z} \cdot r_{z} \cdot C^{\prime-1}\left(r_{z}\right) d z \leq H
\end{array}\right.
$$

The model in (8) can then be modified as follows:

$$
\left\{\begin{array}{l}
L=\operatorname{Max}_{r} \int_{0}^{1}\left[w z+\left(1-\frac{w}{2}\right)\right] \cdot n_{z} \cdot C^{\prime-1}\left(r_{z}\right) d z \\
\text { s.t. } \int_{0}^{1} n_{z} \cdot r_{z} \cdot C^{\prime-1}\left(r_{z}\right) d z=H, n_{z}=M \sum_{j=1}^{N} f_{j}(z)
\end{array}\right.
$$

By the Lagrange method, we can get as follows:

Given the Lagrange multiplier, $\lambda$, which is real number, and the function $r$, where $r=r_{z}$, then

$$
\begin{aligned}
L(\lambda, r) & =\int_{0}^{1}\left[w z+\left(1-\frac{w}{2}\right)\right] \cdot n_{z} \cdot C^{\prime-1}\left(r_{z}\right) d z+\lambda\left[H-\int_{0}^{1} n_{z_{z}} \cdot r_{z} \cdot C^{\prime-1}\left(r_{z}\right) d z\right] \\
& =\int_{0}^{1}\left[\left(w z+\left(1-\frac{w}{2}\right)\right]-\lambda \cdot r_{z}\right) n_{z} \cdot C^{\prime-1}\left(r_{z}\right) d z+\lambda H
\end{aligned}
$$

To obtain an optimal solution for $L^{*}, L^{*}$ must be set to the target value corresponding to $\left(\lambda^{*}, r^{*}\right)$. $\lambda^{*}$ can then be obtained from the following equation

$$
H=\int_{0}^{1} n_{z} \cdot r_{z}\left(\lambda^{*}\right) \cdot C^{\prime-1}\left(r_{z}\left(\lambda^{*}\right)\right) d z \text { and } r_{z}^{*}=r_{z}\left(\lambda^{*}\right)
$$

We know that the optimal value of Lagrange multiplier, $\lambda^{*}$, is correlated with the exogenous variables 
of $w, \mu_{j}, \sigma_{j}^{2}, H, c, N, M$, and hence, the $\lambda^{*}$ can be written as

$$
\lambda^{*}=\lambda^{*}\left(w, \mu_{j}, \sigma_{j}, c, H, M, N\right)
$$

Then we obtain the optimal value of $r_{z}^{*}$ :

$$
\begin{aligned}
r_{z}^{*}\left(w, \mu_{j}, \sigma_{j}, c, H, M, N\right)=\frac{\left[w z+\left(1-\frac{w}{2}\right)\right] \sqrt{2 c H}}{\sqrt{M} \sqrt{w^{2} \sum_{j} \sigma_{j}^{2}+\sum_{j}\left(w \mu_{j}+1-\frac{w}{2}\right)^{2}}} \\
\lambda^{*}=\frac{w z+\left(1-\frac{w}{2}\right)}{\frac{C^{\prime-1}\left(r_{z}^{*}\right)}{C^{\prime-1}\left(r_{z}^{*}\right)}+r_{z}^{*}}, \forall z
\end{aligned}
$$

where $C^{\prime-1}$ is the inverse function of $C^{\prime}$. That is, if $u=C^{\prime}(y)$, then $y=C^{\prime-1}(u)$ and hence $\frac{d y}{d u}=C^{\prime-1}(u)$.

Also, we know that $\frac{\partial r_{z}^{*}}{\partial z}$ has the following properties:

$$
\frac{\partial r_{z}^{*}}{\partial z}=\frac{w \sqrt{2 c H}}{\sqrt{M} \sqrt{\left[w^{2} \sum_{j} \sigma_{j}^{2}+\sum_{j}\left(w \mu_{j}+1-\frac{w}{2}\right)^{2}\right]}}
$$

\section{Measuring the Energy Conservation Performance of an Industry through Energy Values}

Because science cannot deal directly with issues relating to energy resource values, such values must be confirmed in order to compare all possible solutions and identify the optimal solution.

$$
H=\int_{0}^{1} n_{z} \cdot \frac{\left[w z+\left(1-\frac{w}{2}\right)\right]^{2}}{2 \lambda^{*}} \frac{1}{2 c} \frac{1}{2 \lambda^{*}} d z
$$

Then $\lambda^{*}=\frac{|w| \sqrt{M}}{\sqrt{8 c H}} \sqrt{\sum_{j} \sigma_{j}^{2}+\sum_{j}\left(\mu_{j}+\frac{1}{w}-\frac{1}{2}\right)^{2}}$ 
Such that the optimal objective function $L^{*}$ can be written as below:

$$
\begin{aligned}
L^{*} & =\frac{1}{4 c \lambda^{*}} \int_{0}^{1} n_{z}\left[w z+\left(1-\frac{w}{2}\right)\right]^{2} d z=2 H \lambda^{*} \\
& =\sqrt{\frac{H M}{2 c}\left[w^{2}\left(\sum_{j} \sigma_{j}^{2}\right)+\sum_{i}\left(w \mu_{j}+1-\frac{w}{2}\right)^{2}\right]}
\end{aligned}
$$

\section{Conclusions}

For the reason that the government shall need to make sure that all the people and industries can have sufficient energy to use. [6] Of course, all the people on earth have to find and use renewable energy and green energy instead of fossil fuel finally, then our next generation will have enough energy to use. However, people do not cherish the energy. [7] Before we find renewable energy and green energy, we wish the government to do something for us. [8]

In the study, we suggest government should make the measurement into the effect on energy use.

With the measure in the study, that is the energy conservation performance model, we can control the rate energy use and give rewards to the industries. This model we made will serve some responses, it can be applied to the design of an energy performance reward system in which the nation focuses on energy resources with either poorer or higher capacity. [9] This research can also serve a range of purposes, as it can induce nation in the energy conservation performance reward system to not merely focus on industries with either good or bad performance [10].

The results of the study are as follows:

1. The optimal solution for $\lambda^{*}$ will go down as $c$ or $\mathrm{H}$ increase but up as $M, N, \mu_{j}$ or $\sigma_{j}^{2}$ increase.

2. Optimal energy conservation performance $L^{*}$ goes up with $M, N, \mu_{j}$ or $\sigma_{j}$ or $\mathrm{H}$ increases but goes down as $c$ increases.

3. From (12), we can have the following properties as the sensitivity analysis in the study:

If we assume the weight value is a linear function of $w$, then due to $\int_{0}^{1} W_{z} d z=1$

We can get $W_{z}=w \cdot z+\left(1-\frac{w}{2}\right)$. With the properties we can do the sensitivity analysis as follows:

(1). When $w>0, r_{z}^{*}$ is an increasing function of $z$, and its ratio $\left|\frac{\partial r_{z}^{*}}{\partial z}\right|$ will increase as $c$ or $H$ increase, but decreases as $M, N, \mu_{j}$ or $\sigma_{j}^{2}$ increase.

(2). When $w\left\langle 0, r_{z}^{*}\right.$ is a decreasing function of $z$ and its ratio $\left|\frac{\partial r_{z}^{*}}{\partial z}\right|$ will increase as $c$ or $H$ increase, but decreases as $M, N, \mu_{j}$, or $\sigma_{j}^{2}$ increase.

(3). When $w=0, r_{z}^{*}(z)=2 \lambda^{*} \quad \forall z$, We can obtain $\frac{\partial r_{z}^{*}(z)}{\partial z} \equiv 0$. That is, $r_{z}^{*}(z)$ is the constant 
function of $z$.

\section{References}

[1] Chen MS, Yang MY. The concept of contribution theory as a framework of taxation. The Indian Journal of Economics, 1999; 80(316):81-98.

[2] Jencks C, et al., Inequality: A Reassessment of the Effect of Family and Schooling in America. Basic Books, Inc., New York, 1972.

[3] Lazear EP. Teacher incentives. Swedish Economic Policy Review, 2003; 10(2):179-214.

[4] Lazear EP. Performance pay and productivity. American Economic Review, 2000; 90:1346-1361.

[5] Kamien MI, Schwartz NL. Dynamic Optimization: The Calculus of Variations and Optimal Control in Economics and Management. New York: Elsevier North Holland, 1981.

[6] Horne M. A New Role for CTE. Techniques, 2010:10-11.

[7] Hon CC, Guh YY, Wang KM, Lee ES. Fuzzy multiple attributes and multiple hierarchical decision making. Computers Mathematics Applications, 1996; 32(12):109-119.

[8] Kagiwada H, Kalaba RE, Schumitzky A, Sridhar R. Cauchy and fredholm methods for euler equations. Journal of Optimization Theory and Applications. 1968; 2(3):157-163.

[9] Jencks C, Smith M, Acland H, Bane MJ, Cohen D, Gintis H, Heyns B, Michelson S. Inequality, Basic Books, New York, 1972.

[10] Roy S. The Littlest Consumers, Disp Des Ideas. 2004; 16(7):18-19. 